\title{
Preventive role of Propofol in hypoxia/reoxygenation-induced apoptotic H9c2 rat cardiac myoblast cell death
}

\author{
SEONMIN LEE ${ }^{1 *}$, KIYOON KIM $^{1 *}$, YUN HONG KIM ${ }^{3}$, MIN HYUNG CHUNG ${ }^{2}$, \\ INSUG KANG ${ }^{1}$, JOOHUN HA ${ }^{1}$ and WONCHAE CHOE ${ }^{1}$ \\ ${ }^{1}$ Department of Biochemistry and Molecular Biology, Medical Science and Engineering Research Center for Bioreaction to \\ Reactive Oxygen Species and Biomedical Science Institute (BK-21), School of Medicine, Kyung Hee University; \\ ${ }^{2}$ Department of Obstetrics and Gynecology, School of Medicine, Kyung Hee University, Seoul 130-701; \\ ${ }^{3}$ Department of Anesthesiology and Pain Medicine, Kangbuk Samsung Hospital, \\ Sungkyunkwan University School of Medine, Seoul 110-746, Korea
}

Received October 4, 2010; Accepted January 6, 2011

DOI: $10.3892 / \mathrm{mmr} .2011 .432$

\begin{abstract}
Reperfusion of ischaemic myocardium is accompanied by cardiomyocyte apoptosis. Although a protective role of propofol in this process has been suggested, the exact mechanism of propofol activity remains to be elucidated. Here, we report that propofol protects cardiac H9c2 cells from hypoxia/ reoxygenation-induced cell death by attenuating the phosphorylation of extracellular signal-regulated kinases (ERKs) and by up-regulating heme oxygenase 1 (HO-1) expression levels. Treatment with $25 \mu \mathrm{M}$ propofol significantly protected against hypoxia/reoxygenation-induced cell death, as determined by the MTT (3-(4,5-dimethylthiazol-2-yl)-2,5-diphenyltetrazolium bromide) assay and Western blot analysis using anti-apoptotic signal proteins, such as apoptotic protease activating factor 1 and caspase 9. Propofol furthermore suppressed the ERK signaling pathway in cardiac H9c2 cells subjected to hypoxia/ reoxygenation. The up-regulation of anti-oxidant enzymes such as HO-1, manganese superoxide dismutase (MnSOD) and catalase is also associated with the protective effect of propofol on hypoxia/reoxygenation-induced cell death. Taken together, the results reveal a new mechanism by which propofol inhibits hypoxia/reoxygenation-induced cell death in cardiac $\mathrm{H} 9 \mathrm{c} 2$ cells, supporting a potential application of propofol as a preemptive cardioprotectant.
\end{abstract}

\section{Introduction}

Numerous types of cardioprotective therapies following acute myocardial infarction (AMI) have focused on the rapid

Correspondence to: Dr Wonchae Choe, Department of Biochemistry and Molecular Biology, School of Medicine, Kyung Hee University, \#1, Hoegi-dong, Dongdaemoon-gu, Seoul 130-701, Korea

E-mail: wchoe@khu.ac.kr

*Contributed equally

Key words: hypoxia, reoxygenation, propofol, heme oxygenase 1, extracellular signal-regulated kinases restoration of coronary artery blood flow, percutaneous coronary intervention (PCI) or coronary artery bypass grafting (CABG). Although rapid reperfusion has achieved significant success in terms of large-scale reductions in patient mortality and morbidity, reperfusion of the ischaemic myocardium is inevitably accompanied by the loss of myocytes, a pheno menon resulting from lethal reperfusion-induced injury (1). A simple reduction in myocytes decreases the contractile function of the heart and causes the cardiomyocytes to have limited regenerative capacity (2). Reperfusion-induced apoptosis is now established as an important component of cardiac remodeling, particularly in the transition towards overt heart failure (3), and a greater understanding of the apoptotic events in cardiomyocyte apoptosis is imperative. One of the major stimuli for the initiation of apoptosis is ischemia/reperfusion, which occurs during a myocardial infarction or cardiac bypass surgery. Therefore, future therapies must focus on limiting reperfusion-induced apoptosis following AMI in order to increase the salvage of ischaemic myocardium, thus improving mortality and morbidity (4).

Ischemia/reperfusion-induced cell injury after the restoration of oxygen (reoxygenation) is an important event, not only in myocardial infarction and cardiac bypass surgery as mentioned above, but also in other ischemic syndromes. It comprises inflammatory damage caused by the accumulation of free radical oxygen species and triggers the stress signaling process, which eventually results in cell death (5-6). Reactive oxygen species (ROS), including hydrogen peroxide, are generated from mitochondria during ischemia/reperfusion as a consequence of aerobic metabolism. The cellular toxicity of ROS is associated with the rapid modification of cellular constituents, including the depletion of intracellular glutathione and ATP, a decrease in $\mathrm{NAD}^{+}$levels and an increase in free cytosolic $\mathrm{Ca}^{++}$and lipid peroxidation (7). Numerous signaling events stimulated by hypoxia/reoxygenation are mediated by members of the mitogen-activated protein kinase (MAPK) family. The production of ROS in particular triggers the activation of MAPK/ERK kinase-1/2 signaling via the phosphorylation of serine/threonine residues. Furthermore, hypoxic ischemic injury strongly induces HO-1, an ubiqui- 


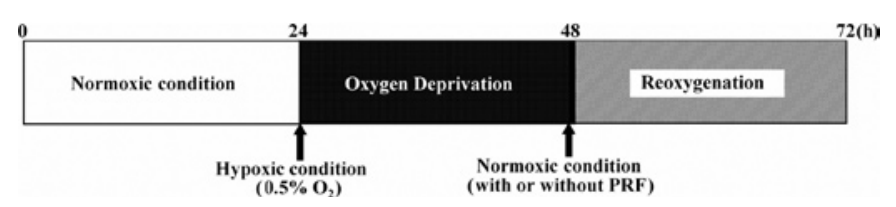

Figure 1. Protocol of in vitro hypoxia/reoxygenation with propofol treatment. $\mathrm{H} 9 \mathrm{c} 2$ cells were exposed to hypoxic conditions (oxygen deprivation, $0.5 \%$ $\mathrm{O}_{2}$ ) for $24 \mathrm{~h}$ without glucose and serum. After hypoxia, cells were reoxygenated under normoxic conditions (reoxygenation) for $24 \mathrm{~h}$. Cells were treated with propofol (PRF) while subjected to reoxygenation.

tous and redox-sensitive inducible stress protein. HO-1 has been revealed to have protective effects against injury. HO-1 induction in donor organs in rats has been revealed to ease ischemia/reperfusion-induced injury, prolong graft survival and improve the long-term function of the grafted kidney (8). Previously, Acquaviva et al indicated that propofol attenuated peroxynitrite-mediated DNA damage and apoptosis by the up-regulation of HO-1 expression (9).

Propofol (2,6-diisopropylphenol), an intravenous anesthetic, has been found to attenuate oxidative stress induced by mechanical and metabolic derangements in the isolated rat heart, and to prevent ischemia/reperfusion injury (10-12). Its antioxidant mechanism is mainly related to its phenolic chemical structure. Previously, it was reported that propofol protects cardiac $\mathrm{H} 9 \mathrm{c} 2$ cells from $\mathrm{H}_{2} \mathrm{O}_{2}$-induced injury by triggering the activation of Akt and a parallel up-regulation of Bcl-2 (13). Furthermore, propofol was reported to protect cardiomyocytes against $\mathrm{H}_{2} \mathrm{O}_{2}$-induced apoptosis, and its antiapoptotic effect was attributed to the increased expression of HO-1 (14). Accordingly, numerious previous studies have demonstrated that propofol contributes to cardioprotection against ischemia-reperfusion injury; however, the mechanism of the protective role of propofol in cardiomyocytes in response to hypoxia/reoxygenation remains unclear. In the present study, we induced apoptosis by hypoxia/reoxygenation, which more closely mimics ischemia/reperfusion injury to cardiomyocytes. We then monitored the protective effects of popofol against ischemia/reperfusion injury by measuring cell viability and the expression levels of apoptotic proteins. Furthermore, we investigated the mechanism of the protective role of propofol against the hypoxia/reoxygenation-induced injury by analyzing ERK signaling and HO-1 expression levels.

\section{Materials and methods}

Materials. Dulbecco's modified Eagle's medium/F-12 (DMEM/F-12) and donor calf serum (CS) were purchased from Gibco-BRL (Grand Island, NY, USA). Propofol (Pofol inj) was provided by Dongkook Pharmaceutical, Co. Ltd. (Korea). Antibody against phospho-ERK was purchased from Upstate Biotechnology Inc. (Lake Placid, NY, USA). Antibodies against ERK, caspase 9, Apaf1, HO-1, Glut1 and actin were purchased from Santa Cruz Biotechnology (Santa Cruz, CA, USA).

Cell culture and assessment of cell viability. H9c2 rat cardiac myoblasts were cultured in proliferation medium composed of DMEM/F12 supplemented with $10 \%$ CS and $1 \%$ antibiotics.

Cell viability was assessed by an MTT reduction conversion assay in 12-well plates. Optical density was assessed at
$550 \mathrm{~nm}$ in a microplate reader (Bio-Rad Laboratories Inc., CA, USA). Cell viability was expressed as the percentage of absorbance relative to that of untreated cells.

In vitro model of hypoxia/reoxygenation and propofol treatment. Cells were exposed to hypoxic conditions (oxygen deprivation, $0.5 \% \mathrm{O}_{2}$ ) for $24 \mathrm{~h}$. After hypoxia, the cells were reoxygenated under normoxic conditions (reoxygenation) for $24 \mathrm{~h}$. Propofol was added to the cells during reoxygenation (Fig. 1).

Western blot analysis. Western blotting was performed as previously described (15). In brief, total lysates were resolved on polyacrylamide gels and blotted onto nitrocellulose membranes. The membranes were washed twice with TBST before the blocking of non-specific binding with $3 \%$ BSA/TBST. The membranes were incubated with primary antibodies for $2 \mathrm{~h}$ at room temperature. After washing, the membranes were incubated with secondary antibodies for $1 \mathrm{~h}$ at room temperature. The membranes were washed five times, followed by detection with horseradish peroxidase-conjugated antibodies for $1.5 \mathrm{~h}$ at room temperature. Results were viewed using enhanced chemiluminescence (ECL) (Amersham, Braunschweig, Germany). For quantification, band intensity was measured by a Bio-Rad Imaging Densitometer (Quantity One software, version 4.6.2).

\section{Results}

Effect of propofol on $\mathrm{H} 9 \mathrm{c} 2$ cell viability during hypoxial reoxygenation. It is well known that hypoxia/reoxygenation induces apoptotic cell death. Therefore, we first established a hypoxia/reoxygenation protocol (Fig. 1). H9c2 cells were reoxygenated under normoxic conditions for $24 \mathrm{~h}$ after $24 \mathrm{~h}$ of hypoxia. In order to determine which concentration of propofol is most effective at preventing hypoxia/reoxygenation -induced cell death, various concentrations of propofol were added to the cells during reoxygenation (Fig. 2A). Cell viability $(\sim 80 \%)$ was observed after the $24 \mathrm{~h}$ of reoxygenation with or without propofol using the MTT assay. Propofol at concentrations of 50 or $250 \mu \mathrm{M}$ did not aid in the recovery of the H9c2 cells from hypoxia/reoxygenation-induced cell death, but rather decreased cell viability by up to $60 \%$. Propofol at $25 \mu \mathrm{M}$ markedly restored cell viability by up to $100 \%$ (Fig. 2A). Although lipopid was used as a solvent control, it had no effect on the results (data not shown). The results indicate that $25 \mu \mathrm{M}$ propofol was effective in protecting cells from oxidative stress in the in vitro hypoxia/ reoxygenation model. Therefore, $25 \mu \mathrm{M}$ propofol was used in the subsequent experiments.

Effect of propofol on hypoxia/reoxygenation-induced cell death through the regulation of ERK phosphorylation in $H 9 c 2$ cells. The apoptosome complex, including apoptotic protease activating factor 1 (Apaf-1) and the activation of caspase, are key steps in the process of apoptosis. Therefore, in order to examine the mechanism of the protective effects of propofol on hypoxia/reoxygenation-induced cell death in $\mathrm{H} 9 \mathrm{c} 2$ cells, the expression of Apaf1 and the cleavage of caspase 9 was analyzed by Western blotting. Glut1 and actin 
A

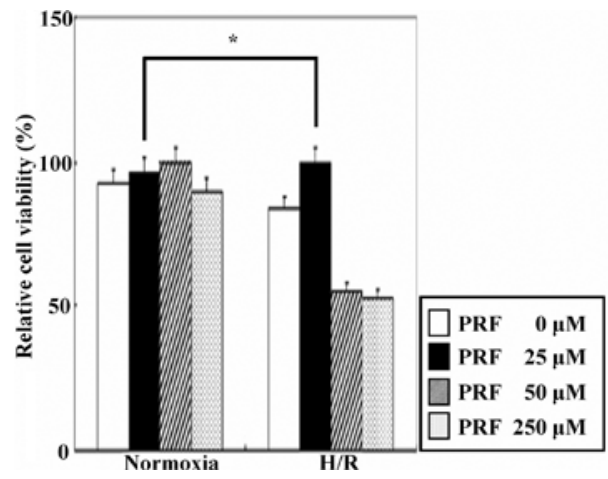

C

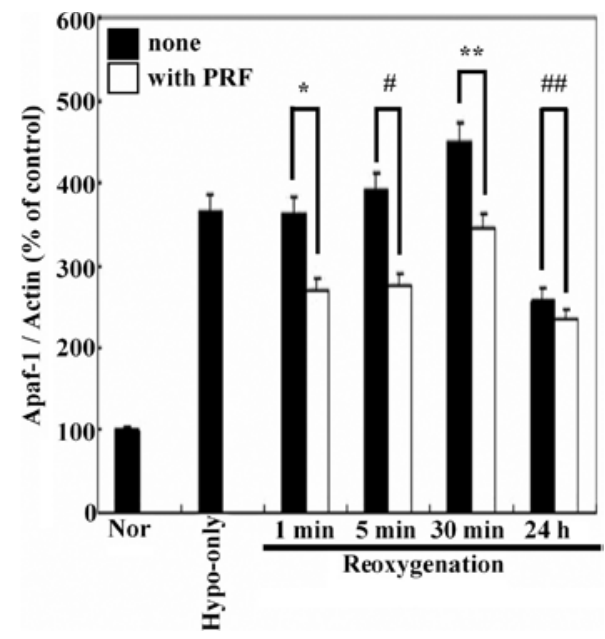

B

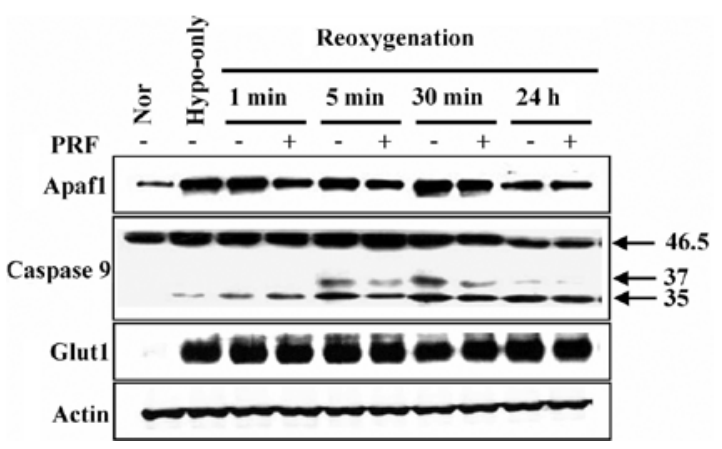

D

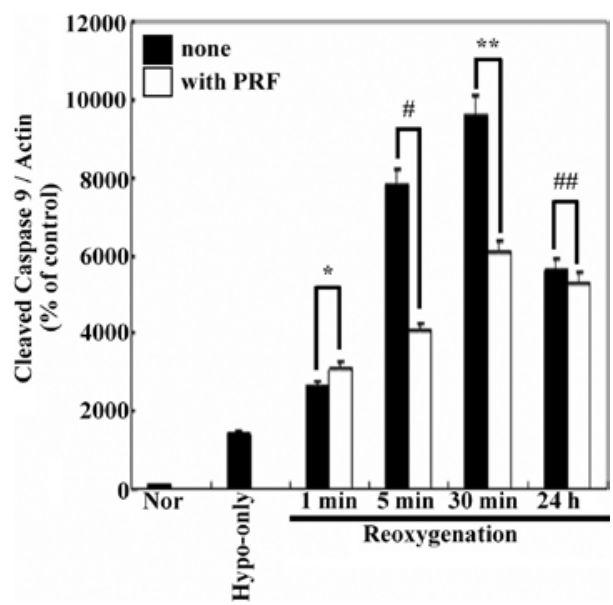

Figure 2. Effect of propofol on H9c2 cell viability during in vitro hypoxia/reoxygenation. (A) H9c2 cells were treated with various concentrations of propofol $(0,25,50$ and $250 \mu \mathrm{M})$ during hypoxia/reoxygenation. Cell viability was measured using the MTT assay. Values are expressed as the means \pm SD of four experiments performed in duplicate. ${ }^{*} \mathrm{P}<0.001$, statistically significant vs. normoxia with $25 \mu \mathrm{M}$ PRF. (B) The expression of Apaf1 and processed caspase 9 was monitored by Western blot analysis at various time points during hypoxia/reoxygenation after treatment with $25 \mu \mathrm{M}$ propofol. Normoxia cells were incubated without hypoxia/reoxygenation. Glut 1 and actin were used as an intrinsic marker of hypoxia and a loading control, respectively. (C) Quantitative analysis of the effects of propofol on Apaf1 expression during in vitro hypoxia/reoxygenation. Values are expressed as the means \pm SD ( $n=3$ ). " $\mathrm{P}<0.05$, ${ }^{\#} \mathrm{P}<0.05,{ }^{* *} \mathrm{P}<0.001$ and ${ }^{\# \#} \mathrm{P}<0.001$ vs. untreated cells. (D) Quantitative analysis of the effects of propofol on caspase 9 processing during in vitro hypoxia/

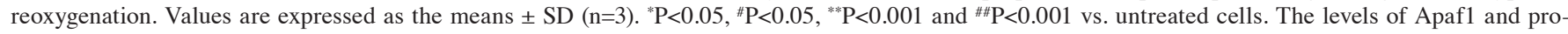
cessed caspase 9 were normalized to the actin level.

were used as an intrinsic marker of hypoxia and a loading control, respectively. Hypoxia increased the expression level of Apaf1 by 3.7-fold, compared to cells under normoxic conditions. As shown in Fig. 2B and C, propofol reduced the expression level of Apaf1 and attenuated caspase 9 cleavage during reoxygenation, as compared to the untreated cells. The protective effects of propofol against hypoxia/reoxygenationinduced Apaf1 expression and caspase 9 cleavage peaked at 27 and 50\%, respectively, 5 min after the initiation of reoxygenation.

In order to determine whether signaling through the ERK signaling pathway mediates the protective effect of propofol on hypoxia/reoxygenation-induced cell death in $\mathrm{H} 9 \mathrm{c} 2$ cells, ERK phosphorylation was measured after treatment with propofol. Propofol was found to supress the phosphorylation of ERK, which was activated during reoxygenation (Fig. 3). At the 30-min time point post reoxygenation, propofol attenuated ERK phosphorylation by up to $55 \%$ compared to the untreated cells. These results suggest that propofol inhibits hypoxia/ reoxygenation-induced cell death through the ERK signaling pathway.
Effect of propofol on the expression of anti-oxidant enzymes during hypoxia/reoxygenation-induced cell death. In order to determine whether the regulation of anti-oxidant enzymes is involved in the protective function of propofol against hypoxia/reoxygenation, the expression levels of HO-1, MnSOD and catalase were monitored in the H9c2 cells during hypoxia/reoxygenation by Western blot analysis (Fig. 4). Hypoxia alone as well as reoxygenation significantly increased the expression level of HO-1. Treatment with propofol markedly increased HO-1 expression, with significance up to the 30-min time point after reoxygenation, compared to the untreated cells. By contrast, at the 24-h time point after reoxygenation, there were no significant differences between the cells treated with or without propofol. MnSOD and catalase were significantly induced by propofol at 1 and 5 min after reoxygenation compared to the untreated cells. These results suggest that the up-regulation of anti-oxidant enzymes, such as HO-1, MnSOD and catalase, may be associated with the protective function of propofol against apoptotic cell death caused by hypoxia/reoxygenation-induced oxidative stress via ERK signal regulation. 
A

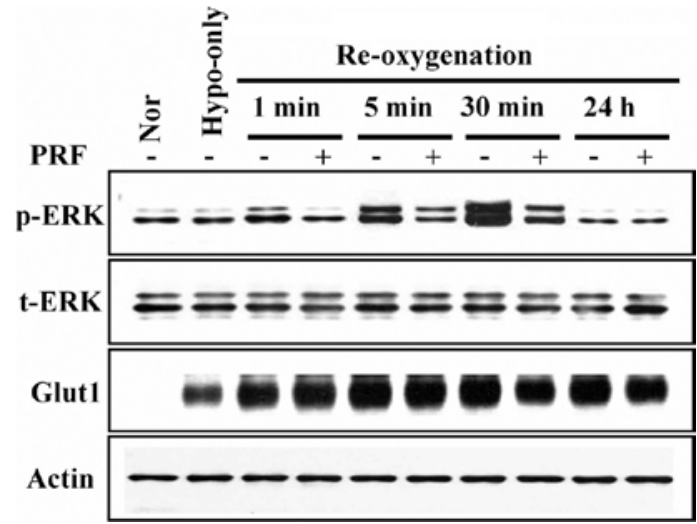

B

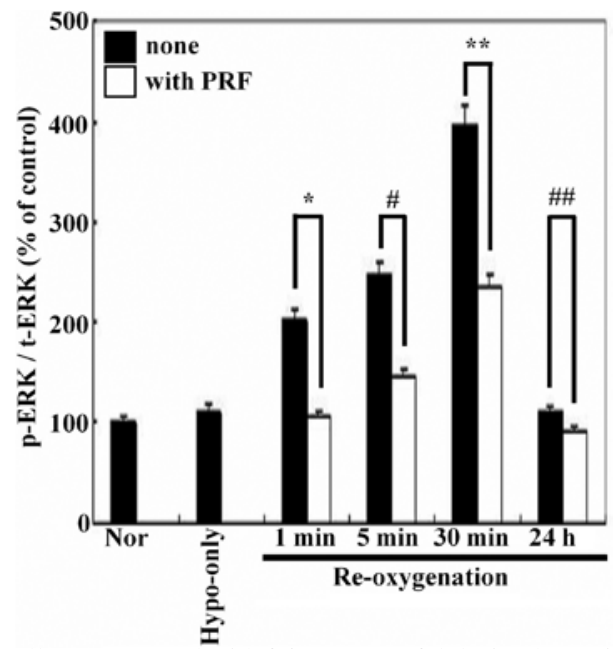

Figure 3. Effect of propofol on ERK phosphorylation during hypoxia/reoxygenation. H9c2 cells were treated with $25 \mu \mathrm{M}$ propofol during hypoxia/reoxygenation and cell lysates were harvested. (A) Verification of ERK phosphorylation by Western blot analysis. Normoxia cells were incubated without hypoxia/ reoxygenation. Glut 1 and actin were used as an intrinsic marker of hypoxia and a loading control, respectively. (B) Quantitative analysis of ERK phosphorylation. Each signal was quantified by densitometric scanning. Values are expressed as the means \pm SD of three replicate measurements in three different cell cultures. The phosphorylation level of ERK was normalized to the level of total ERK. ${ }^{*} \mathrm{P}<0.05,{ }^{\#} \mathrm{P}<0.05,{ }^{* *} \mathrm{P}<0.05$ and ${ }^{\# \#} \mathrm{P}<0.001$ vs. untreated cells.
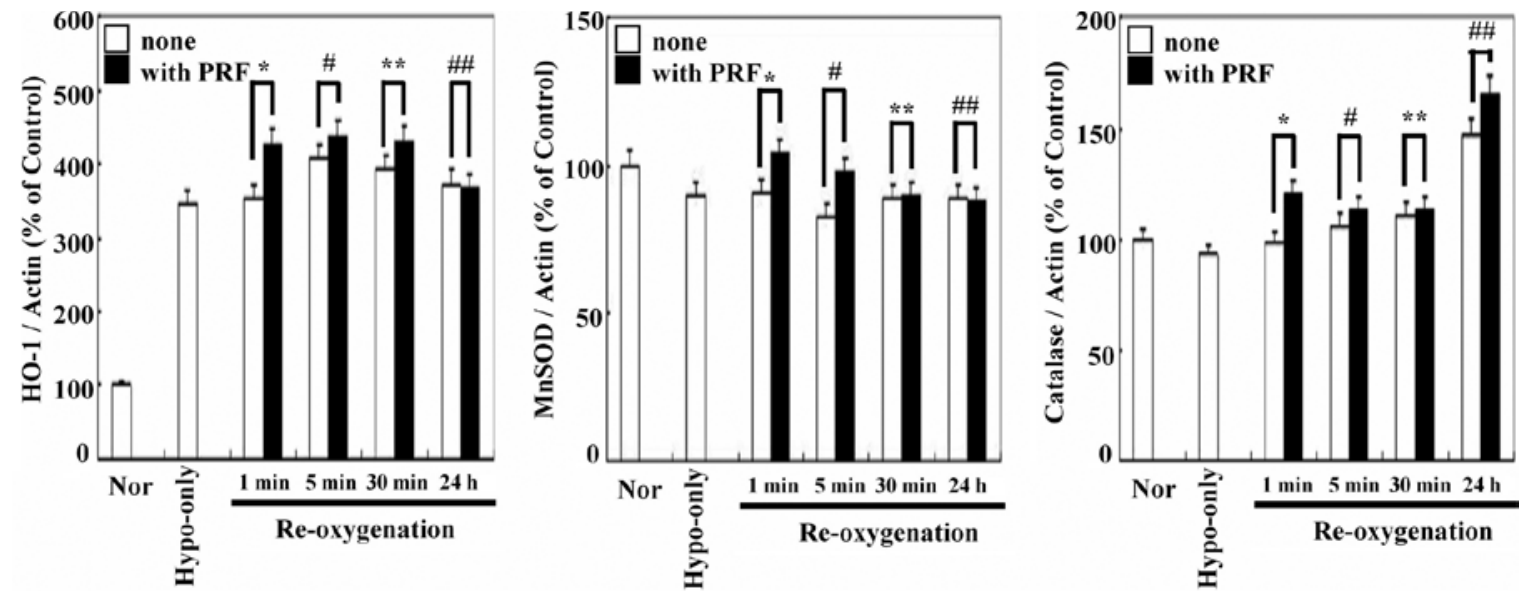

Figure 4. Altered expression of anti-oxidant enzymes in $\mathrm{H} 9 \mathrm{c} 2$ cells with propofol treatment during hypoxia/reoxygenation. The expression levels of the antioxidant enzymes HO-1, MnSOD and catalase were determined by Western blot analysis at various points during hypoxia/reoxygenation after treatment with $25 \mu \mathrm{M}$ propofol. Normoxia cells were incubated without hypoxia/reoxygenation. Glut 1 and actin were used as an intrinsic marker of hypoxia and a loading control, respectively. Quantitative analysis of anti-oxidant enzyme expression levels was conducted. Each signal was quantified by densitometric scanning. Values are expressed as the means \pm SD of three replicate measurements in three different cell cultures. The expression levels of anti-oxidant enzymes were normalized to the actin level. ${ }^{*} \mathrm{P}<0.05,{ }^{\#} \mathrm{P}<0.001,{ }^{* *} \mathrm{P}<0.001$ and ${ }^{\# *} \mathrm{P}<0.001$ vs. untreated cells.

\section{Discussion}

In this study, we revealed the protective effect of propofol in a hypoxia/reoxygenation model, as determined by the MTT assay and the evaluation of Apaf1 expression and caspase-9 activation. Moreover, we found that the protective effect of propofol against hypoxia/reoxygenation-induced apoptotic cell death is associated with the ERK signaling pathway and the induced expression of anti-oxidant enzymes, such as HO-1, MnSOD and catalase.

A previous study reported that propofol had no cardioprotective effect against ischemia/reperfusion injury at a concentration of up to $10 \mu \mathrm{M}$ (16). Elsewhere, it was revealed that propofol provided a dose-dependent protection of cardiac function (12). Furthermore, $1 \mu \mathrm{M}$ propofol was found to confer sufficient protection against hypoxia/reoxygenation injury in rat cardio- myocytes, while propofol concentrations above $1 \mu \mathrm{M}$ exhibited negative effects on cell viability (12). Our results demonstrate that $25 \mu \mathrm{M}$ propofol was effective, while 50 or $250 \mu \mathrm{M}$ propofol had deleterious effects on cell viability. Therefore, it is possible that a dose-dependent difference may exist depending on the experimental protocols and systems. However, we demonstrated a cardioprotective effect of propofol against ischemia/ reperfusion injury consistent with previous findings.

ERK, a member of the MAPK family, is known to be a signal of the cardiac reperfusion injury salvage kinase (RISK) pathway. When activated in the setting of cardiac ischaemia/ reperfusion, the ERK cascade plays an important role in preventing cellular apoptosis (17-18). Our results indicate that hypoxia/reoxygenation injury activated ERK signaling and decreased cell viability in rat cardiomyocytes, while propofol attenuated the elevated ERK signaling and maintained cell 
viability in cells exposed to hypoxia/reoxygenation. These findings suggest an essential role of ERK signaling in propofol activity. By contrast, previous studies found that propofol activated ERK signaling for protection against hypoxia/ reoxygenation (19). However, other lines of evidence are in accordance with our results, indicating that propofol attenuates ERK signaling. Propofol pre-treatment revealed cytoprotection through the attenuation of lipopolysachharide-induced phosphorylation of ERK (20). Furthermore, propofol inhibited the phosphorylation of ERK induced by ANG II via a decrease in ROS production (21). Nagata et al found that propofol inhibits ERK activation in human neutrophils (22). Kozinn et al found that propofol eliminated the glutamatergic activation of the ERK pathway in the hippocampus neurons (23). The exact relationship of ERK phosphorylation with propofol remains controversial. The discrepancy between whether propofol activates or attenuates the ERK signaling in cells exposed to hypoxia/reoxygenation requires further study using other hypoxia/reoxygenation experimental systems.

The up-regulation of HO-1 after exposure to hypoxia/ reoxygenation has previously been reported in various cell lines (24). Most stimuli of HO-1 gene induction occur on the transcriptional level and are mediated by a variety of cis-regulatory elements localized in the HO-1 promoter (25). A prominent role for the up-regulation of HO-1 under hypoxic conditions has been ascribed to the transcription factor HIF-1 $\alpha$. Another transcription factor that may participate in HO-1 induction is NRF2, particularly in a stress-dependent manner. It has been reported that HO-1 expression is related to the anti-apoptotic effect of propofol on cultured astrocytes treated with a cytotoxic agent (9). Propofol protected rat kidneys against reperfusion injury by increasing $\mathrm{HO}-1$ activity (26). Our results indicate that propofol at the clinically available dosage of $25 \mu \mathrm{M}$ had an anti-apoptotic effect on reoxygenated $\mathrm{H} 9 \mathrm{c} 2$ cells through the up-regulation of HO-1. This is consistent with the findings of previous studies, which revealed that appropriate propofol concentrations significantly increased HO-1 expression (9). It has also been demonstrated that HO-1 gene expression is regulated by ERK signaling, which is also consistent with our data. Recently, similar observations regarding the induction of HO-1 gene expression by hypoxia/reoxygenation were reported for a model of hypoxia/ reoxygenation in cultured cardiomyocytes (27).

Notably, as shown in Fig. 4, a clinical dose of propofol significantly enhanced HO-1 expression for up to $30 \mathrm{~min}$ post-reoxygenation, compared to the untreated cells. However, there were no differences in HO-1 expression at $24 \mathrm{~h}$ postreoxygenation. These results suggest that an early phase of HO-1 regulation is important for recovery, which is supported by our results regarding Apaf1 and caspase 9 expression (Fig. 2B-D) and the ERK signaling pathway (Fig. 3A and B). In addition, propofol treatment led to the significant induction of MnSOD and catalase in hypoxia/reoxygenation injury, which indicates an increased capacity of propofol to handle free radicals and other oxidants leading to cell survival.

In summary, a clinical dose of propofol protects H9c 2 cells against reoxygenation injury. The protective effect of propofol may be mediated by the activation of antioxidant enzymes, such as HO-1, MnSOD and catalase, which is modulated by upstream ERK signaling.

\section{Acknowledgements}

This study was supported by the Program of Kyung Hee University for Young Researchers in Medical Science 2008 (KHU20081235).

\section{References}

1. Lo HM, Kloner RA and Braunwald E: Effect of intracoronary verapamil on infarct size in the ischemic, reperfused canine heart: critical importance of the timing of treatment. Am J Cardiol 56: 672-677, 1985.

2. Dubois G, Segers VF, Bellamy V, et al: Self-assembling peptide nanofibers and skeletal myoblast transplantation in infarcted myocardium. J Biomed Mater Res B Appl Biomater 87: 222-228, 2008.

3. Regula KM and Kirshenbaum LA: Apoptosis of ventricular myocytes: a means to an end. J Mol Cell Cardiol 38: 3-13, 2005.

4. Mudalagiri NR, Mocanu MM, Di Salvo C, et al: Erythropoietin protects the human myocardium against hypoxia/reoxygenation injury via phosphatidylinositol-3 kinase and ERK1/2 activation. Br J Pharmacol 153: 50-56, 2008.

5. Fliss $\mathrm{H}$ and Gattinger D: Apoptosis in ischemic and reperfused rat myocardium. Circ Res 79: 949-956, 1996.

6. Anaya-Prado R and Toledo-Pereyra LH: The molecular events underlying ischemia/reperfusion injury. Transplant Proc 34: 2518-2519, 2002.

7. Navarro A and Boveris A: The mitochondrial energy transduction system and the aging process. Am J Physiol Cell Physiol 292: C670-C686, 2007.

8. Tullius SG, Nieminen-Kelha M, Buelow R, et al: Inhibition of ischemia/reperfusion injury and chronic graft deterioration by a single-donor treatment with cobalt-protoporphyrin for the induction of heme oxygenase-1. Transplantation 74: 591-598, 2002.

9. Acquaviva R, Campisi A, Murabito P, et al: Propofol attenuates peroxynitrite-mediated DNA damage and apoptosis in cultured astrocytes: an alternative protective mechanism. Anesthesiology 101: 1363-1371, 2004.

10. Kokita N and Hara A: Propofol attenuates hydrogen peroxideinduced mechanical and metabolic derangements in the isolated rat heart. Anesthesiology 84: 117-127, 1996.

11. Xia Z, Godin DV and Ansley DM: Propofol enhances ischemic tolerance of middle-aged rat hearts: effects on $15-\mathrm{F}(2 \mathrm{t})$ isoprostane formation and tissue antioxidant capacity. Cardiovasc Res 59: 113-121, 2003.

12. Xia Z, Godin DV, Chang TK and Ansley DM: Dose-dependent protection of cardiac function by propofol during ischemia and early reperfusion in rats: effects on 15-F2t-isoprostane formation. Can J Physiol Pharmacol 81: 14-21, 2003.

13. Wang B, Shravah J, Luo H, Raedschelders K, Chen DD and Ansley DM: Propofol protects against hydrogen peroxide-induced injury in cardiac $\mathrm{H} 9 \mathrm{c} 2$ cells via Akt activation and Bcl-2 up-regulation. Biochem Biophys Res Commun 389: 105-111, 2009.

14. $\mathrm{Xu} \mathrm{JJ}$ and Wang YL: Propofol attenuation of hydrogen peroxide-mediated oxidative stress and apoptosis in cultured cardiomyocytes involves haeme oxygenase-1. Eur J Anaesthesiol 25: 395-402, 2008.

15. Ding Y, Choi KJ, Kim JH, et al: Endogenous hydrogen peroxide regulates glutathione redox via nuclear factor erythroid 2-related factor 2 downstream of phosphatidylinositol 3-kinase during muscle differentiation. Am J Pathol 172: 1529-1541, 2008.

16. Kurokawa H, Murray PA and Damron DS: Propofol attenuates beta-adrenoreceptor-mediated signal transduction via a protein kinase C-dependent pathway in cardiomyocytes. Anesthesiology 96: 688-698, 2002.

17. Hausenloy DJ and Yellon DM: New directions for protecting the heart against ischaemia-reperfusion injury: targeting the reperfusion injury salvage kinase (RISK)-pathway. Cardiovasc Res 61: 448-460, 2004.

18. Zang WJ, Sun L and Yu XJ: Cardioprotection of ischemic postconditioning and pharmacological post-treatment with adenosine or acetylcholine. Sheng Li Xue Bao 59: 593-600, 2007.

19. Kim HS, Chang WC, Hwang KC, Choi IG and Park WK: Effect of propofol on calcium homeostasis in hypoxia-reoxygenated neonatal rat cardiomyocytes. Eur J Pharmacol 594: 139-145, 2008. 
20. Jawan B, Kao YH, Goto S, et al: Propofol pretreatment attenuates LPS-induced granulocyte-macrophage colony-stimulating factor production in cultured hepatocytes by suppressing MAPK/ERK activity and NF-kappaB translocation. Toxicol Appl Pharmacol 229: 362-373, 2008.

21. Zou XJ, Yang L and Yao SL: Propofol depresses angiotensin IIinduced cardiomyocyte hypertrophy in vitro. Exp Biol Med 233: 200-208, 2008

22. Nagata T, Kansha M, Irita K and Takahashi S: Propofol inhibits FMLP-stimulated phosphorylation of p42 mitogen-activated protein kinase and chemotaxis in human neutrophils. $\mathrm{Br} \mathrm{J}$ Anaesth 86: 853-858, 2001.

23. Kozinn J, Mao L, Arora A, Yang L, Fibuch EE and Wang JQ: Inhibition of glutamatergic activation of extracellular signalregulated protein kinases in hippocampal neurons by the intravenous anesthetic propofol. Anesthesiology 105: 1182-1191, 2006.
24. Mitani K, Fujita H, Sassa S and Kappas A: Activation of heme oxygenase and heat shock protein 70 genes by stress in human hepatoma cells. Biochem Biophys Res Commun 166: 1429-1434, 1990.

25. Ohlmann A, Giffhorn-Katz S, Becker I, Katz N and Immenschuh S: Regulation of heme oxygenase-1 gene expression by anoxia and reoxygenation in primary rat hepatocyte cultures. Exp Biol Med 228: 584-589, 2003.

26. Wang HH, Zhou HY, Chen CC, Zhang XL and Cheng G: Propofol attenuation of renal ischemia/reperfusion injury involves heme oxygenase-1. Acta Pharmacol Sin 28: 1175-1180, 2007.

27. Foresti R, Goatly H, Green CJ and Motterlini R: Role of heme oxygenase-1 in hypoxia-reoxygenation: requirement of substrate heme to promote cardioprotection. Am J Physiol Heart Circ Physiol 281: H1976-H1984, 2001. 